\title{
The Magnetic Fields of the Planets: A New Scaling Law of the Dipole Moments of the Planetary Magnetism
}

\author{
Yasuharu SANO \\ Department of Geophysics, Faculty of Science, Kyoto University, Kyoto 606-01, Japan
}

(Received April 6, 1992; Revised November 19, 1992)

\begin{abstract}
A new scaling law for the planetary magnetism was developed on the assumption that the Lorentz force balances the Coriolis force in the planetary core. In deriving the scaling law we decomposed the vector fields into the toroidal and the poloidal components, and dealt with them separately. From the scaling law, not only we can predict the dipole moments of the planets, but also can we estimate the toroidal magnetic field intensity in the planetary core; the result implies a typical toroidal magnetic field intensity of $100[\mathrm{G}]$, and the toroidal and the poloidal velocity fields respectively of the orders of $1 \times 10^{-5}\left[\mathrm{~ms}^{-1}\right]$ and $4 \times 10^{-7}\left[\mathrm{~ms}^{-1}\right]$ in the Earth's core. Since the present study is based on an $\alpha \omega$-dynamo model, the resultant scaling law depends on the efficiency of the $\alpha$-effect. If we adopt the dependence of the form $\alpha \propto \Omega$ (with $\Omega$ the angular velocity of the planet's self-rotation), the magnetic dipole moment $M$ of a planet scales as $M \propto(\text { characteristic length })^{7 / 2}$ (mean density $)^{1 / 2}$ (angular velocity). The predictions agree well with the observations except Venus and Mars.
\end{abstract}

\section{Introduction}

Observations have revealed that a number of cosmic bodies possess their intrinsic magnetic fields. BLACKETT (1947), was the first who discussed the existence of a scaling law (or a magnetic Bode's law) for the magnetic fields of cosmic bodies. He proclaimed that the magnetic dipole moment of a cosmic body scaled as its own angular momentum. He argued that a rotating body, even if electrically neutral, gave rise to magnetic field through some unknown unification theory of gravity and electromagnetism. Now it is already accepted that the intrinsic magnetic fields of cosmic bodies, including those of the planets, are due to the dynamo action operative in the electrically conducting fluid core of the cosmic bodies (e.g. textbooks of MoFFATT, 1978; JACOBS, 1987). Although some workers have denied the existence of scaling laws in the opinion that each planet should have each dynamo, there are also several workers who relied on its possibility and have attempted to deduce or to test scaling laws for the planetary magnetism based on the dynamo theory (Busse, 1976; Russell, 1978, 1979; JACOBS, 1979). Different may be the type of dynamo from planet to planet, we still recognize a possibility of existence of some kind of relation between the magnetic fields of the planets if any type of dynamo at the planets can be finally attributed to the same type of basic equations. Although a simple order estimation can by no means replace the full solutions (which may be dependent on the actual type of dynamo at each planet), it has an advantage that it can provide a rough estimation of the physical quantities without getting into any details of the actual dynamos of the planets. CURTIS and NESS (1986) derived a scaling law starting with torque balance between Coriolis and Lorentz forces (Magnetostrophic balance). Two different scaling laws were presented by MizUTANI et al. (1992) also by assuming the magnetostrophic balance. Most of the scaling laws above were derived by treating the vector equations in the scalar form.

In this paper we decompose incompressible vector fields into the toroidal and the poloidal components in order to improve the scalar treatment, and derive a scaling law of the planetary 
magnetism treating these two components separately. This approach also allows us to estimate the toroidal magnetic field intensity, which is unobservable from outside the planet's core. Of course the estimation of the toroidal magnetic field intensity is dependent on what one employs as the model. For instance, BRAGINSKII $(1975,1980)$ constructed a nearly symmetric dynamo model on the basis of magnetostrophic balance, and predicted very strong toroidal magnetic field in the Earth's core (Strong field model). On the other hand, Busse (1976) adopted the geostrophic balance, namely, a balance between Coriolis and pressure gradient forces, and made an estimation that the toroidal magnetic field was of the same order of magnitude as that of the poloidal magnetic field (Weak field model). Thus, the estimation of the toroidal magnetic field intensity in the planetary core is closely related to the type of torque balance one assumes in the planetary core. The results presented in this paper favors the magnetostrophic balance rather than the geostrophic balance in the planetary core, giving the toroidal magnetic field intensity of by one order of magnitude larger than that of the poloidal field for the Earth. At the same time, we suggest that this ratio is not invariant among the planets; it depends on several physical parameters and hence can vary from planet to planet.

\section{Derivation of a Scaling Law}

\subsection{Toroidal and poloidal decomposition}

The turbulent dynamo problem is described by the following mean-field MHD equations:

$$
\begin{aligned}
\frac{\partial \mathbf{B}}{\partial t} & =\operatorname{rot}(\mathbf{v} \times \mathbf{B}+\alpha \mathbf{B})+\frac{1}{\mu \sigma} \nabla^{2} \mathbf{B} \\
\rho\left\{\frac{\partial \mathbf{v}}{\partial t}+(\mathbf{v} \cdot \nabla) \mathbf{v}\right\} & =-\nabla p+\frac{1}{\mu} \operatorname{rot} \mathbf{B} \times \mathbf{B} \\
& \quad+2 \rho \mathbf{v} \times \mathbf{\Omega}+(\text { Buoyancy, Gravity, etc. }) \\
\operatorname{div} \mathbf{v}= & 0 .
\end{aligned}
$$

Here $\mathbf{B}$ is the magnetic field, $\mathbf{v}$ velocity field, $\mu$ permeability, $\sigma$ electric conductivity, $\rho$ density of the planetary core, $p$ pressure, and $\boldsymbol{\Omega}$ angular velocity of the planetary core. The term $\alpha \mathbf{B}$ in Eq. (1) expresses the mean electromotive force arising from interactions between turbulent velocity and magnetic fields (STEEnBECK et al., 1966). The terms $\overline{\left(\mathbf{v}^{\prime} \cdot \nabla\right) \mathbf{v}^{\prime}}$ and $\overline{\operatorname{rot} \mathbf{B}^{\prime} / \mu \times \mathbf{B}^{\prime}}$, with $\mathbf{v}^{\prime}$ and $\mathbf{B}^{\prime}$ fluctuations of $\mathbf{v}$ and $\mathbf{B}$ and an overline expressing some appropriate average, might be important in some cases, but these terms are neglected in Eq. (2) for simplicity. We have also assumed incompressible fluid and no kinetic viscosity. Given a divergence-free vector field a, we can introduce the toroidal-poloidal decomposition of this vector field by

$$
\mathbf{a}=\operatorname{rot}(\Psi \mathbf{r})+\operatorname{rot} \operatorname{rot}(\Phi \mathbf{r})
$$

where $\Psi$ and $\Phi$ are appropriate scalar functions. The first term denotes a toroidal field and the second term a poloidal field (see appendix. See also MoffatT, 1978). We then decompose the velocity field and the magnetic field in the core of a planet into their toroidal and poloidal components:

$$
\mathbf{v}=\mathbf{v}_{T}+\mathbf{v}_{P}, \quad \mathbf{B}=\mathbf{B}_{T}+\mathbf{B}_{P} .
$$

Here the subscript ${ }_{T}$ refers to the toroidal component and the subscript ${ }_{P}$ to the poloidal component, respectively. The equation of continuity (3) is automatically satisfied by the velocity field of the form (4). If convective cells are formed in the planetary core, the non-axisymmetric modes of the poloidal velocity field will also become important, but the average contributions from those modes can be included in the turbulent electromotive force term $\alpha \mathbf{B}_{T}$ and $\alpha \mathbf{B}_{P}$ as 
small deviations from axisymmetry (BRAGINSKII, 1975). Thus, for the axisymmetric part, we write down the toroidal and the poloidal parts of the induction and the Navier-Stokes equations as

$$
\begin{aligned}
\frac{\partial \mathbf{B}_{T}}{\partial t}= & \operatorname{rot}\left(\mathbf{v}_{T} \times \mathbf{B}_{P}+\mathbf{v}_{P} \times \mathbf{B}_{T}+\alpha \mathbf{B}_{P}\right)+\frac{1}{\mu \sigma} \nabla^{2} \mathbf{B}_{T}, \\
\frac{\partial \mathbf{B}_{P}}{\partial t}= & \operatorname{rot}\left(\mathbf{v}_{P} \times \mathbf{B}_{P}+\alpha \mathbf{B}_{T}\right)+\frac{1}{\mu \sigma} \nabla^{2} \mathbf{B}_{P}, \\
\rho\left\{\frac{\partial \mathbf{v}_{T}}{\partial t}+\right. & \left.\left(\mathbf{v}_{T} \cdot \nabla\right) \mathbf{v}_{P}+\left(\mathbf{v}_{P} \cdot \nabla\right) \mathbf{v}_{T}\right\} \\
= & \frac{1}{\mu} \operatorname{rot} \mathbf{B}_{T} \times \mathbf{B}_{P}+2 \rho \mathbf{v}_{P} \times \mathbf{\Omega}, \\
\rho\left\{\frac{\partial \mathbf{v}_{P}}{\partial t}+\right. & \left.\left(\mathbf{v}_{T} \cdot \nabla\right) \mathbf{v}_{T}+\left(\mathbf{v}_{P} \cdot \nabla\right) \mathbf{v}_{P}\right\} \\
= & -\nabla p+\frac{1}{\mu}\left(\operatorname{rot} \mathbf{B}_{P} \times \mathbf{B}_{P}+\operatorname{rot} \mathbf{B}_{T} \times \mathbf{B}_{T}\right) \\
& +2 \rho \mathbf{v}_{T} \times \mathbf{\Omega}+(\text { Buoyancy, Gravity, etc. }) .
\end{aligned}
$$

In the following analysis, we will study the case when the characteristic time scale $\tau$ of the dynamo action is sufficiently longer than the diffusion time of the magnetic field in the planetary core. This allows us to omit the time-derivatives in (6) and (7). In (8) and (9) also, the time-derivatives can be neglected since $\tau \gg \Omega^{-1}$. The $\varphi$-component of the pressure gradient is omitted in the toroidal component of the equation of motion (8), since $\partial / \partial \varphi \equiv 0$ in the axisymmetric configuration.

\subsection{Estimation of order of magnitude}

We now estimate the order of magnitude of each equation. In (6), we will first assume that $v_{T} B_{P} \gtrsim v_{P} B_{T}$. We will also assume the $\alpha \omega$-mechanism of the dynamo. In the formulation of an $\alpha \omega$-dynamo problem, the poloidal part of the mean-electromotive force $\alpha \mathbf{B}_{P}$ is neglected compared with the $\omega$-effect term. We then find from (6) that

$$
\frac{1}{\ell} v_{T} B_{P} \sim \frac{1}{\mu \sigma} \frac{1}{\ell^{2}} B_{T} \gg \frac{B_{T}}{\tau}
$$

i.e.

$$
B_{T} \sim \mu \sigma \ell v_{T} B_{P} .
$$

All the values are those for the planet's core, and $\ell$ is the characteristic length of the conducting region of the planetary interior such as the core radius. Similarly, Eq. (8) leads to

$$
B_{P} B_{T} \sim 2 \mu \rho \ell v_{P} \Omega \text {. }
$$

Let us take the magnetostrophic balance also in the poloidal component of equation of motion (9). This yields the following estimation:

$$
(\nabla p \lesssim)\left|\frac{1}{\mu}\left(\operatorname{rot} \mathbf{B}_{P} \times \mathbf{B}_{P}+\operatorname{rot} \mathbf{B}_{T} \times \mathbf{B}_{T}\right)\right| \sim 2 \rho v_{T} \Omega
$$

The range of the Lorentz force can be estimated as:

$$
\begin{aligned}
& \left\{\max \left(B_{T}^{2}, B_{P}^{2}\right)-\min \left(B_{T}^{2}, B_{P}^{2}\right)\right\} / \mu \ell \\
& \lesssim\left|\frac{1}{\mu}\left(\operatorname{rot} \mathbf{B}_{P} \times \mathbf{B}_{P}+\operatorname{rot} \mathbf{B}_{T} \times \mathbf{B}_{T}\right)\right| \\
& \lesssim\left\{\max \left(B_{T}^{2}, B_{P}^{2}\right)+\min \left(B_{T}^{2}, B_{P}^{2}\right)\right\} / \mu \ell .
\end{aligned}
$$


With the average of the left and the right hand sides as the characteristic order of magnitude of the middle hand side, we find

$$
\left|\frac{1}{\mu}\left(\operatorname{rot} \mathbf{B}_{P} \times \mathbf{B}_{P}+\operatorname{rot} \mathbf{B}_{T} \times \mathbf{B}_{T}\right)\right| \sim\left\{\begin{array}{lll}
B_{P}^{2} / \mu \ell & \text { for } & \left(B_{P} \gg B_{T}\right) \\
B_{P}^{2} / \mu \ell \sim B_{T}^{2} / \mu \ell & \text { for } & \left(B_{P} \sim B_{T}\right) \\
B_{T}^{2} / \mu \ell & \text { for } & \left(B_{P} \ll B_{T}\right) .
\end{array}\right.
$$

Hence we obtain

$$
2 \mu \rho \ell v_{T} \Omega \sim B_{P}^{2} \quad \text { for } \quad\left(B_{P} \gtrsim B_{T}\right)
$$

and

$$
2 \mu \rho \ell v_{T} \Omega \sim B_{T}^{2} \quad \text { for } \quad\left(B_{P} \lesssim B_{T}\right)
$$

Although we do not totally exclude the possibility of the case $B_{P} \gg B_{T}$, we think it is unlikely, for it requires an extremely efficient $\alpha$-effect to maintain a steady magnetic field. With the more probable cases of $B_{P} \sim B_{T}$ and $B_{P} \ll B_{T}$ (which can be unified as $B_{P} \lesssim B_{T}$ ), we find from Eqs. (10), (11), and (16) that

$$
\begin{aligned}
v_{T} & \sim \frac{2 \rho \Omega}{\mu \ell\left(\sigma B_{P}\right)^{2}}, \\
B_{T} & \sim \frac{2 \rho \Omega}{\sigma B_{P}} \\
v_{P} & \sim \frac{1}{\mu \sigma \ell} .
\end{aligned}
$$

Expressions (17) and (19) lead to

$$
\frac{B_{T}}{B_{P}} \sim \frac{v_{T}}{v_{P}}\left(\sim \frac{2 \rho \Omega}{\sigma B_{P}^{2}}\right) .
$$

On the other hand, if we assume that $v_{T} B_{P} \lesssim v_{P} B_{T}$ in Eq. (6), Eq. (10) should be replaced by

$$
B_{T} \sim \mu \sigma \ell v_{P} B_{T}
$$

which again leads to the same $v_{P}$ as given in (19), and hence to the same $v_{T}$ and $B_{T}$ as derived from Eq. (6). Thus we find that $v_{T} B_{P} \sim v_{P} B_{T}$ holds with the expressions of $v_{T}, B_{T}$, and $v_{P}$ given by $(17),(18)$, and (19), respectively. The result (19) implies that the poloidal (i.e. convective) velocity in the planetary core remains of the order of the diffusion velocity of magnetic field. This is due to the back-reaction from the magnetic field; if the poloidal velocity field enhances by chance, it will induce larger toroidal magnetic field which, through back-reaction, acts to squeeze the increased poloidal velocity field. In the steady state, therefore, a poloidal velocity field of the order of the magnetic diffusion velocity shall be realized.

The ratio of Lorentz force to Coriolis force is measured by the Elsasser number (ELSASSER, 1946) defined by

$$
E \ell=\frac{\sigma B^{2}}{\rho \Omega}
$$

In the same manner we can introduce a dimensionless number

$$
E \ell_{P}=\frac{\sigma B_{P}^{2}}{\rho \Omega} \sim \frac{\left|\sigma\left(\mathbf{v}_{P} \times \mathbf{B}_{P}\right) \times \mathbf{B}_{P}\right|}{\left|\rho \mathbf{v}_{P} \times \mathbf{\Omega}\right|} \sim \frac{\left|\mathbf{j}_{T} \times \mathbf{B}_{P}\right|}{\left|\rho \mathbf{v}_{P} \times \mathbf{\Omega}\right|}
$$


which may be termed as the poloidal Elsasser number. Using this dimensionless number, the ratio $B_{T} / B_{P}$ is expressed as $B_{T} / B_{P} \sim v_{T} / v_{P} \sim 2 / E \ell_{P}$, and the condition $B_{P} \lesssim B_{T}$ can be written as $E \ell_{P} \lesssim 1$ (the factor of 2 was omitted).

For the Earth we use the following values for the parameters:

$$
\begin{aligned}
& \mu \sim 1.3 \times 10^{-6}\left[\mathrm{NA}^{-2}\right], \quad \sigma \sim 5 \times 10^{5}\left[\mathrm{Sm}^{-1}\right], \\
& \rho \sim 1 \times 10^{4}\left[\mathrm{kgm}^{-3}\right], \quad \Omega \sim 7.3 \times 10^{-5}\left[\mathrm{~s}^{-1}\right], \\
& \ell \sim 3.5 \times 10^{6}[\mathrm{~m}], \quad B_{P} \sim 3 \times 10^{-4}[\mathrm{~T}] .
\end{aligned}
$$

A little higher value of the electric conductivity of the core was used than was given in HoNKURA and Matsushima (1988). The estimated values are

$$
\begin{aligned}
& v_{P} \sim 4 \times 10^{-7}\left[\mathrm{~ms}^{-1}\right], \quad v_{T} \sim 1 \times 10^{-5}\left[\mathrm{~ms}^{-1}\right], \\
& B_{T} \sim 1 \times 10^{-2}[\mathrm{~T}], \quad E \ell_{P} \sim 0.062, \quad \frac{B_{T}}{B_{P}} \sim 32 .
\end{aligned}
$$

The toroidal magnetic field intensity in the Earth's core, estimated above, is by one order of magnitude larger than that of the poloidal magnetic field. To be noted here is that the velocity field derived above are somewhat smaller than the values presented in the previous works both based on frozen-flux approximation (MUTH and BENTON, 1981; MADDEN and LEMouËL, 1982; GIRE et al., 1986; VoORHIERs, 1986) and based on $\alpha \omega$-mechanism (RIKITAKE, 1967; MATSUSHIMA and HoNKURA, 1988, 1989). One of the reasons for this disagreement may be the incorporation of the back-reaction of the magnetic field which we take into account. Also the assumption of steady solution may have led to underestimate.

\subsection{Scaling law for the magnetic fields of the planets}

We have so far dealt with $B_{P}$ as one of the known parameters, and have expressed $v_{T}, v_{P}$, and $B_{T}$ using basic parameters including $B_{P}$. Next, we try to give a scaling law for $B_{P}$ of the planets. From the remaining equation (7) we obtain

$$
B_{P} \sim \mu \sigma \ell \alpha B_{T}
$$

The term $\mathbf{v}_{P} \times \mathbf{B}_{P}$ is omitted since this term is known to make no contribution to the dynamo action in the axisymmetric configuration (COWLING, 1934; BRAGINSKII 1964). With the relation (10) and the $B_{T}$ given by (16), we find

$$
\alpha \sim \frac{1}{v_{T}(\mu \sigma \ell)^{2}} \sim \frac{B_{P}^{2}}{2 \mu \rho \ell \Omega} .
$$

By substitution of appropriate numerical values for the Earth we estimate that $\alpha \sim 1 \times 10^{-8}\left[\mathrm{~ms}^{-1}\right]$ in the Earth's core. Since the coefficient $\alpha$ reflects the characteristics of turbulent, small-scale motions in the core, it is assumed here that $\alpha$ is not directly dependent on the large-scale parameters such as $\ell, v_{T}, v_{P}$, except on $\Omega$ which, through Coriolis force acting on the small-scale motions, may give rise to non-vanishing helicity which would generate the mean electromotive force of the form $\alpha \mathbf{B}_{T}$ (PARKer, 1955; Steenbeck and Krause, 1969; see also Moffatt, 1978). And since we have obtained by now no direct information on the characteristic scales (spatial and temporal) of the turbulent motions in the planetary core, we have nothing but to assume also that those scales coincide for every planet. If we always take $\Omega$ as positive, we can take $\alpha$ as a function of 
$\Omega$ like $\alpha=\alpha(\Omega)$, rather than a function of $|\Omega|$. And if $\alpha(\Omega)$ vanishes at $\Omega=0$, the leading term in the Taylor-series expansion of $\alpha$ with respect to $\Omega$ leads to $\alpha \propto \Omega$, i.e.

$$
\frac{\alpha^{P}}{\alpha^{E}}=\frac{\Omega^{P}}{\Omega^{E}}
$$

where the superscripts ${ }^{P}$ and ${ }^{E}$ refer to the planetary and the terrestrial values, respectively. For the characteristic dipole moment $M$ defined by

$$
M \sim B_{P} \cdot \ell^{3} \sim \ell^{3} \sqrt{2 \mu \rho \ell \alpha \Omega}
$$

we derive a scaling law:

$$
\frac{M^{P}}{M^{E}}=\left(\frac{\ell^{P}}{\ell^{E}}\right)^{7 / 2}\left(\frac{\rho^{P}}{\rho^{E}}\right)^{1 / 2}\left(\frac{\Omega^{P}}{\Omega^{E}}\right) .
$$

Since the expression (25) does not involve the electric conductivity $\sigma$ of the planet's core, the scaling law (28) also becomes independent of $\sigma$, provided that the system can be regarded as being steady in time, and that the relation (20) holds. Of course the result should be modified if the mean electromotive force $\alpha$ depends on the electric conductivity $\sigma$. However, KRAUSE and RÄDLER (1980) have shown that the coefficient $\alpha$ does not depend on $\sigma$ when the diffusion time is sufficiently longer than the correlation time of the small-scale turbulence. Therefore we regard that the coefficient $\alpha$ is approximately independent of the electric conductivity in the planet's core.

\section{Discussion}

\subsection{Results of the wcaling law}

To compare the dipole moments of the planets, it is necessary to know the values of the characteristic length $\ell$ and the mean density $\rho$ for the conducting region of each planet. For the terrestrial planets we assume that the core mean density is the same as that of the Earth. For the giant planets, we take the mean density in the conducting region as 4.0, 2.5, 4.0, and $5.0\left[\times 10^{3} \mathrm{kgm}^{-3}\right]$ respectively for Jupiter, Saturn, Uranus, and Neptune (NESS, 1978; PHILLIPS and MAlin, 1983; TSERKLEvich et al., 1983; Stevenson, 1982; GudKova et al., 1988; HuBBerd et al., 1991). For the terrestrial planets we take $\ell$ equal to the core radius. The giant planets have central rocky core which, as opposed to the Earth's inner core, is thought to be electrically insulating. In this case the scaling law should be

$$
M \sim\left(\ell_{\text {out }}-\ell_{\text {in }}\right)^{1 / 2} \ell_{\text {out }}^{3} \rho^{1 / 2} \Omega
$$

where $\ell_{\text {out }}$ is the core radius and $\ell_{\text {in }}$ the radius of the central rocky core, $\ell_{\text {out }}-\ell_{\text {in }}$ being the radial extent of the metallic hydrogen/ice region. The values of the fundamental parameters normalized to the Earth's values are listed in Table 1 together with the values of the non-dimensional parameter $E \ell_{P}$. From the top, radius of the central rocky core $\ell_{\text {in }}$, core radius $\ell_{\text {out }}$, core mean density $\rho$, angular velocity $\Omega$, dimensionless number $E \ell_{P}$, toroidal/poloidal ratio $\gamma \equiv 2 / E \ell_{P}$, logarithm of the dipole moments of the planetary magnetic field based on observations, and based on the present scaling law, respectively. A common $\sigma$ of $5 \times 10^{5}\left[\mathrm{Sm}^{-1}\right]$ for every planet was assumed during the calculation of $E \ell_{P}$ in this table. Using these parameters, the predicted dipole moments of the planets are plotted against the dipole moments determined from observations in Fig. 1.

The dipole moments of the planets are based on the spacecraft observations (NESS, 1979; Phillips and Russell, 1987; Connerney, 1981; Connerney et al., 1982; Connerney et al., 1987; Ness et al., 1989). Upper limits are shown for Venus and Mars. The thick line of $45^{\circ}$ 
Table 1. The planetary parameters normalized to the Earth's values, and the values of the dimensionless number $E \ell_{P} \equiv \sigma B_{P}^{2} / \rho \Omega$ and $\gamma \equiv 2 / E \ell_{P} . E \ell_{P}$ and $\gamma$ are not listed for Venus and Mars. Radii of rocky core of the giant planets are given in units of Earth's core radius.

\begin{tabular}{rcccccccc}
\hline & $\mathrm{Me}$ & $\mathrm{V}$ & $\mathrm{E}$ & $\mathrm{Ma}$ & $\mathrm{J}$ & $\mathrm{S}$ & $\mathrm{U}$ & $\mathrm{N}$ \\
\hline Radius of rocky core & - & - & - & - & 4.1 & 4.3 & 2.2 & 1.4 \\
Core radius & 0.53 & 0.93 & 1.00 & 0.47 & 16 & 8.7 & 5.3 & 4.3 \\
Core mean density & 1.0 & 1.0 & 1.0 & 1.0 & 0.40 & 0.25 & 0.40 & 0.50 \\
Angular velocity & 0.017 & 0.004 & 1.000 & 0.972 & 2.409 & 2.246 & 1.537 & 1.299 \\
$E \ell_{P}$ & 0.002 & - & 0.062 & - & 5.5 & 0.22 & 0.2 & 0.03 \\
$\gamma \equiv 2 / E \ell_{P}$ & 1000 & - & 32 & - & 0.36 & 9.0 & 10 & 62 \\
$\log M$ (observed) & -3.30 & $-4.98 \dagger$ & 0.00 & $-3.60 \dagger$ & 4.27 & 2.76 & 1.69 & 1.40 \\
$\log M$ (predicted) & -2.8 & -2.5 & 0.0 & -1.1 & 4.3 & 3.2 & 2.4 & 2.1 \\
\hline
\end{tabular}

( $†$ upper limits)

declination shows predictions coinciding with observations. We recognize in the figure that the scaling law gives satisfactory agreement with the observations in spite of the present uncertainty on the internal structure of the planets other than the Earth. The exception is the cases of Venus and Mars, which are supposed to be inactive in dynamo operation (CURTIS and NESS, 1986). Although the assumption of axisymmetry seems to be too crude for the very eccentric dipoles at Uranus and Neptune, the predicted values are still in good agreement with the observations. However, a closer inspection of the figure shows that there is a tendency of overestimation for the predictions of the scaling law compared with the observations, which is probably because the electric currents do not necessarily extend over the whole planetary core in the strict sense, and this may have led to some overestimate of the value of $\ell_{\text {out }}-\ell_{\text {in }}$. This tendency seems more relevant for Uranus and Neptune, which may be due to their eccentricity of the dipole locations.

There has been a criticism against the existence of scaling laws that a scaling law which is not explicitly dependent on the strength of the driving force is questionable from a physical point of view, for the activity of a dynamo should be inevitably related to some kind of energy supply. The scaling law of CURTIS and NESS (1986) may have been evaluated with this criticism taken into account. The present analysis is based on an idea that a scaling law which does not depend explicitly on the strength of the driving force may be also possible for non-linear MHD dynamos. The energy supply should exceed the required minimum energy to maintain a dynamo against ohmic loss. The magnetic field, in the author's opinion, can grow only until some saturation level if the dynamo is of a non-linear MHD type. And if the rate of energy supply exceeds the Joule loss rate only by a small amount, this saturation level may change sensitively with the changes in the parameters. This means that the Lorentz force and the driving force nearly balances in this case. However, if the rate of energy supply is sufficiently greater than the rate of energy consumption at dynamo, the saturation level may be set at a level which is not critically dependent on the changes in the value of the parameters any more. Only a part of energy input is converted to the energy of magnetic field; other remaining part of the energy input has nothing to do with the activity of dynamo. Presumably, what balances the Lorentz force is not the driving force itself, but Coriolis force for this type of dynamo. If this is the case, a scaling law may be acceptable which does not involve some parameters concerning the energy input explicitly.

As is listed in Table 1 , the condition $E \ell_{P} \lesssim 1$ seems to be true for the planets other than Jupiter, It is, however, unlikely that this condition holds in the case of Jupiter which, if $\sigma \gtrsim 1 \times$ $10^{5}\left[\mathrm{Sm}^{-1}\right]$, gives $E \ell_{P} \gtrsim 1$, and contradicts the assumption $B_{P} \lesssim B_{T}$. One possibility is that 


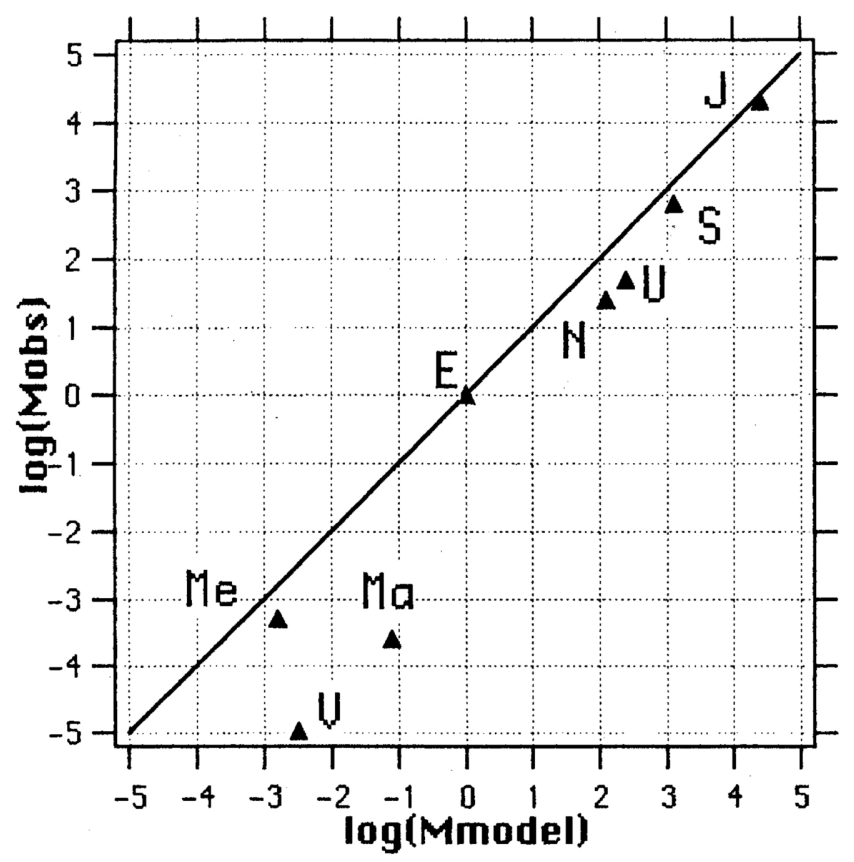

Fig. 1. The predicted planetary dipole moments against the dipole moments determined from observations. Abbreviations used are Me:Mercury, V: Venus, E: Earth, Ma: Mars, J: Jupiter, S: Saturn, U: Uranus, and N: Neptune. Upper limits are shown for Venus and Mars.

$B_{P} \gtrsim B_{T}$ is realized in the Jovian core. Another (and maybe more probable) possibility is that other forces such as buoyancy or pressure gradient become important in the Jovian core as well as the Lorentz and the Coriolis forces (may be termed as a 'supermagnetostrophic balance').

Thus, $E \ell_{P}$ is found to be one of the factors which determine the kind of dynamo - MHD or kinematic. From the definition of $E \ell_{P}$ (Lorentz force/Coriolis force), it might be thought that a non-linear MHD dynamo would be set up when $E \ell_{P}>1$. We, however, take the opposite interpretation; Lorentz force dominates Coriolis force since the dynamo is kinematic. Some kind of driving mechanism such as thermal or compositional convection is required to maintain a dynamo. If the driving force dominates over both the Lorentz and the Coriolis forces (a kinematic dynamo), then the poloidal velocity will exceed the diffusion velocity of the magnetic field, will twist the magnetic field lines strongly beyond the equilibrium between the Lorentz and the Coriolis forces, and will generate stronger magnetic fields than in the MHD case. For those kinematic dynamos, the generated magnetic field is approximately a linear functional of the velocity field. So, if the Lorentz force is a square functional of the velocity field, $E \ell_{P}$ giving the ratio of Lorentz force to Coriolis force becomes approximately proportional to the magnitude of the velocity field. This implies a relation $\mid$ driving force $|>|$ Lorentz force $|\gtrsim|$ Coriolis force $\mid$, which leads to $E \ell_{P} \gtrsim 1$ for kinematic dynamos of this kind. In the opposite case $\mid$ driving force $|<|$ Coriolis force $\mid$, the magnetic field can grow only until the Lorentz force balances the Coriolis force, so we will have $E \ell_{P} \lesssim 1$. This means that the magnetic field settles to its saturation level in a nonlinear MHD case. The poloidal velocity field is suppressed by the magnetic field to the order of magnitude of the diffusion velocity of the magnetic field. For these dynamos, the power of Coriolis force integrated over the source region is of the same order of magnitude as the Joule loss rate of the 
Table 2. Comparison between the several major scaling laws. $M$ denotes the dipole moment, $L$ planetary angular momentum, $\rho$ core mean density, $\ell$ core radius, $\Omega$ angular velocity of the planet, $E$ latent heat flux, $\sigma$ electric conductivity of the core, $v$ characteristic velocity in the core, and $R_{O}$ Rossby number.

\begin{tabular}{cc}
\hline BLACKett (1947) & $M \sim L$ \\
BUSSE (1976) & $M \sim \rho^{1 / 2} \ell^{4} \Omega$ \\
SteVENSON (1983) & $M \sim\left\{\begin{array}{l}\rho^{1 / 2} \ell^{3} \Omega^{1 / 2} \sigma^{-1 / 2} \\
\rho^{1 / 2} v \ell^{3} R_{O}^{-1 / 2}\end{array}\right.$ \\
CURTIS and NESS (1986) & $M \sim \rho^{1 / 3} \ell^{7 / 2} \Omega^{1 / 2} E^{1 / 6}$ \\
ISAS-1 (1992) & $M \sim \rho^{1 / 2} \ell^{7 / 2} \Omega^{3 / 4} \sigma^{-1 / 4}$ \\
ISAS-2 (1992) & $M \sim \rho^{1 / 2} \ell^{3} \Omega^{1 / 2} \sigma^{-1 / 2}$ \\
Present study & $M \sim \rho^{1 / 2} \ell^{7 / 2} \Omega$ \\
\hline
\end{tabular}

energy of the magnetic field:

$$
2 \rho v_{T} v_{P} \Omega \cdot \frac{4 \pi}{3} \ell^{3} \sim \frac{\frac{1}{2 \mu} B_{T}^{2} \times \frac{4 \pi}{3} \ell^{3}}{\mu \sigma \ell^{2}}
$$

which is estimated as $\sim 8 \times 10^{8}[\mathrm{~W}]$ for the Earth.

In the kinematic case where $E \ell_{P} \gtrsim 1$, the driving forces such as buoyancy may need an explicit treatment as opposed to the previous MHD case. In such a kinematic case, the velocity field is first determined from the equation of motion independent of the magnetic field, and then the magnetic field is solved regarding the velocity field as given. Dynamos of such kind are investigated by a lot of workers (for detail, see MOFFATT, 1978; KRAUSE and RÄDLER, 1980). The regular polarity reversals of the solar magnetic field (MAUNDER, 1913; HALE and NICHOLSON, 1925) are known to be well reproduced by the $\alpha \omega$-dynamo model (STEENBECK and KRAUSE, 1969). On the other hand, the two-disk dynamo model (RIKITAKE, 1958) and the associated models (e.g. SHIMIZU and HonKURA, 1986) imply that a highly nonlinear feature appears when the effect of Lorentz force is involved. Hence irregular polarity reversals are expected for an MHD dynamo. If the dimensionless number $E \ell_{P}$ specifies the kind of dynamos, this parameter can be taken as expressing the nature of polarity reversals of the concerned dynamo; for $E \ell_{P} \gtrsim 1$ regular reversals, and for $E \ell_{P} \lesssim 1$ irregular reversals. It may be possible in this sense that the magnetic field at Jupiter would experience rather regular polarity reversals while irregular reversals for other planets.

\subsection{Comparison with other scaling laws}

Table 2 lists several major scaling laws proposed by far. Most of the scaling laws agrees with the observations to a certain extent, but not completely. Unfortunately, it seems still difficult to judge between those scaling laws due to the present uncertainties on the internal structure of the planetary cores. Except $\Omega$, which is very precisely known for every planet, the parameters still suffer great uncertainties. Even the most "primitive" scaling law, (dipole moment) versus (angular momentum), roughly gives a proportionality. Differences between the predictions and the observations seem relatively larger for the scaling law of CURTIS and NESS (1986) than for other scaling laws. Close to the present scaling law is the scaling law of Busse (1976) $M \propto \rho^{1 / 2} \ell^{4} \Omega$. 
If we assume the same electric conductivity for every planet, ISAS-1 scaling law (MizUTANI et al., 1992) $M \propto \rho^{1 / 2} \ell^{7 / 2} \Omega \sigma^{-1 / 4}$ also becomes close to the present scaling law, although the derivation of those scaling laws is very different from the ours. Busse's scaling law gives a good fit to the fields of the terrestrial planets except Venus and Mars (in any scaling law model Venus and Mars do not seem to agree with prediction), but it would give a considerable overestimation to the fields of the giant planets even if the original scaling law were modified as

$$
M \propto\left(\ell_{\text {out }}-\ell_{\text {in }}\right) \ell_{\text {out }}^{3} \Omega .
$$

ISAS-1 scaling law also gives a good prediction to the fields of the planets (except Venus and Mars) provided that the electric conductivity of the core coincides for every planet. Present scaling law gives on the average a good fit both to the fields of the terrestrial and of the giant planets without special requirements on the electric conductivity of the planetary core.

\section{Conclusion}

A scaling law for the planetary magnetism is proposed. We adopt a steady $\alpha \omega$-dynamo model, and assume the magnetostrophic balance both for the poloidal and the toroidal parts of equation of motion. We also assume that the mean electromotive force arising from turbulence in the planetary core is dependent on the angular velocity $\Omega$ of the planet's rotation through a relation $\alpha \propto \Omega$. Based on the present scaling law, the dipole moment $M$ of a planet is given by the combination of three basic parameters $\rho, \ell$, and $\Omega$; the planetary dipole moment scales as $M \propto \ell^{7 / 2} \rho^{1 / 2} \Omega$. The predictions based on the scaling law agree well with the observations except Venus and Mars, which are thought to have no dynamo. It is also found that the state of the whole system is specified by the value of a dimensionless parameter $E \ell_{P}$ defined by $E \ell_{P} \equiv \sigma B_{P}^{2} / \rho \Omega$. When $E \ell_{P} \lesssim 1$, the system is in a magnetostrophic balance, i.e. the balance between Lorentz and Coriolis forces would hold. In this case, the ratio of the toroidal magnetic field to the poloidal magnetic field is estimated to be $2 E \ell_{P}^{-1}$, which is also roughly equal to the ratio of the toroidal velocity field to the poloidal velocity field. In case $E \ell_{P} \gtrsim 1$, the system may be in a supermagnetostrophic balance in which Coriolis force alone cannot sustain Lorentz force. The scaling law suggests a possibility of supermagnetostrophic balance in the Jovian core.

The author is grateful to Prof. T. Araki and Prof. T. Terasawa for useful comments and valuable suggestions. Special thanks are expressed to the two referees for their helpful comments which greatly improved the original manuscript.

\section{APPENDIX}

Let $\mathbf{B}$ be a three dimensional vector field of position and time satisfying $\operatorname{div} \mathbf{B}=0$. Any of its vector potentials be denoted by $\mathbf{A}_{0}$. On the spherical polar coordinates $(r, \theta, \varphi), \mathbf{A}_{0}$ is expressed as

$$
\mathbf{A}_{0}=A_{0 r} \mathbf{e}_{r}+A_{0 \theta} \mathbf{e}_{\theta}+A_{0 \varphi} \mathbf{e}_{\varphi}
$$

where $\mathbf{e}_{i}(i=r, \theta, \varphi)$ denotes the unit vector in the direction of increasing $i$. Toroidal field and poloidal field are the vector fields of the form of $\operatorname{rot}(\Psi \mathbf{r})$ and $\operatorname{rot} \operatorname{rot}(\Phi \mathbf{r})$, respectively. This implies that the vector potential can be taken as having the form of

$$
\mathbf{A}=\Psi \mathbf{r}+\operatorname{rot}(\Phi \mathbf{r})=(\Psi r) \mathbf{e}_{r}+\frac{1}{\sin \theta} \frac{\partial \Phi}{\partial \varphi} \mathbf{e}_{\theta}-\frac{\partial \Phi}{\partial \theta} \mathbf{e}_{\varphi}
$$

However, $\mathbf{A}_{0}$, taken arbitrary, does not generally have the form of (A.2). 
There exists an arbitrariness of the gradient of a scalar function $f$ for the vector potential. We then introduce a new vector potential $\mathbf{A}$ by

$$
\begin{aligned}
\mathbf{A} & =\mathbf{A}_{0}+\nabla f \\
& =\left(A_{0 r}+\frac{\partial f}{\partial r}\right) \mathbf{e}_{r}+\left(A_{0 \theta}+\frac{1}{r} \frac{\partial f}{\partial \theta}\right) \mathbf{e}_{\theta}+\left(A_{0 \varphi}+\frac{1}{r \sin \theta} \frac{\partial f}{\partial \varphi}\right) \mathbf{e}_{\varphi} \\
& \equiv A_{r} \mathbf{e}_{r}+A_{\theta} \mathbf{e}_{\theta}+A_{\varphi} \mathbf{e}_{\varphi} .
\end{aligned}
$$

The condition on this vector potential $\mathbf{A}$ to have the the same form as in (A.2) is

$$
\frac{\partial}{\partial \theta}\left(\sin \theta A_{\theta}\right)+\frac{\partial}{\partial \varphi}\left(A_{\varphi}\right)=0
$$

which can be rewritten as

$$
\triangle_{2} f=-\operatorname{div}_{2} \mathbf{A}_{0}
$$

where

$$
\triangle_{2} f \equiv \frac{1}{r^{2} \sin \theta}\left[\frac{\partial}{\partial \theta}\left(\sin \theta \frac{\partial f}{\partial \theta}\right)+\frac{1}{\sin \theta} \frac{\partial^{2} f}{\partial \varphi^{2}}\right]
$$

and

$$
\operatorname{div}{ }_{2} \mathbf{A}_{0} \equiv \frac{1}{r \sin \theta}\left[\frac{\partial}{\partial \theta}\left(\sin \theta A_{0 \theta}\right)+\frac{\partial A_{0 \varphi}}{\partial \varphi}\right]
$$

It is probably without problem to assume that the right hand side of (A.5) is expandable into a series of spherical harmonics $Y_{n}^{m}(\theta, \varphi)$ in dealing with the vector potentials of the planetary magnetic field:

$$
\text { R.H.S. of }(\mathrm{A} .5) \equiv \sum u_{n}(r) Y_{n}^{m}(\theta, \varphi) .
$$

Equation (A.5) guarantees the existence of the scalar function

$$
f=\sum \frac{r u_{n}(r)}{n(n+1)} Y_{n}^{m}(\theta, \varphi)
$$

as long as the right hand side of (A.5) is expandable into a series of spherical harmonics.

Defining

$$
\Phi \equiv-\int_{0}^{\theta}\left(a_{0 \varphi}+\frac{1}{r \sin \theta} \frac{\partial f}{\partial \varphi}\right) d \theta=\int_{0}^{\varphi}\left(a_{0 \theta}+\frac{1}{r} \frac{\partial f}{\partial \theta}\right) d \varphi
$$

and

$$
\Psi \equiv \frac{1}{r}\left(a_{0 r}+\frac{\partial \Phi}{\partial r}\right)
$$

the vector potential $\mathbf{A}$ is expressed as

$$
\mathbf{A}=\Psi \mathbf{r}+\operatorname{rot}(\Phi \mathbf{r})
$$

which gives

$$
\mathbf{B}=\operatorname{rot}(\Psi \mathbf{r})+\operatorname{rot} \operatorname{rot}(\Phi \mathbf{r})
$$




\section{REFERENCES}

BlaCkett, P. M. S., The magnetic field of massive rotating bodies, Nature, 159, 658-666, 1947.

Braginskil, S. I., Self-excitation of a magnetic field during the motion of a highly-conducting fluid, J.E.T.P., 20, 726-735, 1964.

BraginskiI, S. I., Nearly axially symmetric model of the hydrodynamic dynamo of the Earth I, Geomag. Aeron., , 15, 122-128, 1975.

Braginskil, S. I., Magnetic waves in the core of the Earth II, Geophys. Astrophys. Fluid. Dyn., 14, 189-208, 1980.

Busse, F. H., Generation of planetary magnetism by convection, Phys. Earth Planet. Inter., 12, 350-358, 1976.

Connerney, J. E. P., The magnetic field of Jupiter: a generalized inverse approach, J. Geophys. Res., 86, 7679-7693, 1981.

Connerney, J. E. P., N. F. Ness, and M. H. ACUÑA, Zonal harmonic model of Saturn's magnetic field from Voyager 1 and 2 observations, Nature, 298, 44-46, 1982.

Connerney, J. E. P., M. H. ACuña, and N. F. Ness, The magnetic field of Uranus, J. Geophys. Res., 92, 15329-15336, 1987.

Cowling, T. G., The magnetic fields of sunspots, Mon. Not. Roy. Astron. Soc., 94, 39-48, 1934.

CurTis, S. A. and N. F. NESS, Magnetostrophic balance in planetary dynamos: predictions for Neptune's magnetosphere, J. Geophys. Res., 91, 11003-11008, 1986.

ElSASSER, W. M., Induction effects in terrestrial magnetism II. The secular variation, Phys. Rev., 70, 202--212, 1946.

Gire, C., J. L. LeMoü̈L, and T. MAdDen, Motions at the core surface derived from SV data, Geophys. J. Roy. Astron. Soc., 84, 1-29, 1986.

Gudkova, T. V., V. N. Zharkov, and V. V. LeOnT'Ev, Models of Uranus and Neptune with partially mixed envelopes, Sol. Syst. Res., 22, 23-40, 1988.

HAle, G. E. and S. B. Nicholson, The law of sun-spot polarity, Astrophys. J., 62, 270-300, 1925.

HonkURA, Y. and M. MATSUShima, Lower bound for the electrical conductivity of the Earth's outer core, Geophys. Res. Lett., 15, 689-692, 1988.

Hubberd, W. B., W. J. Nellis, A. C. Mitchell, N. C. Holmes, S. S. Limaye, and P. C. McCandless, Interior structure of Neptune: comparison with Uranus, Science, 253, 648-651, 1991.

JACOBS, J. A., Planetary magnetic fields, Geophys. Res. Lett., 6, 213, 1979.

JACOBS, J. A., Geomagnetism, Vol. 2., Academic Press, 1987.

Krause, F. and K. H. RÄDler, Mean-Field Magnetohydrodynamics and Dynamo Theory, Pergamon Press, 1980.

MAdDEn, T. and J. L. LeMouëL, The recent secular variation and the motions at the core surface, Phil. Trans. Roy. Soc. Lond., 360, 271-280, 1982.

Matsushima, M. and Y. Honkura, Fluctuation of the standing and the drifting parts of the Earth's magnetic field, Geophys. J., 92, 35-50, 1988.

Matsushima, M. and Y. Honkura, Large-scale field motion in the Earth's outer core estimated from non-dipole magnetic field data, J. Geomag. Geoelectr., 41, 963-1000, 1989.

Maunder, E. W., Distribution of sunspots in heliographic latitude, 1874-1913, Mon. Not. Roy. Astr. Soc., 74, $112-116,1913$.

Mizutani H., T.Yамамото, and A.Fujimura, Prediction of magnetic field strength of Neptune: scaling laws of planetary magnetic fields, Adv. Space Res., 12, 265-279, 1992.

Moffatt, H. K., Magnetic Field Generation in Electrically Conducting Fluids, Cambridge University Press, New York, 1978.

Muth, L. A. and E. R. Benton, On the frozen flux velocity field at the surface of the Earth's core necessary to account for the poloidal main magnetic field and its secular variation, Phys. Earth Planet. Inter., 24, 245-252, 1981.

NESS, N. F., Mercury: Magnetic field and structure, Sp. Sci. Revs., 21, 527-554, 1978.

Ness, N. F., The magnetic fields of Mercury, Mars, and Moon, Ann. Rev. Earth Planet. Sci., 7, 249-288, 1979.

Ness, N. F., M. H. Acuña, L. F. Burlaga, J. E. P. Connerney, R. P. Lepping, and F. M. Neubauer, Magnetic fields at Neptune, Science, 246, 1473-1478, 1989.

Parker, E. N., Hydromagnetic dynamo models, Astrophys. J., 122, 293-314, 1955.

Phillips, R. J. and M. C. MAlin, The interior of Venus and tectonic implications, in Venus, pp. 165-167, University of Arizona press, Tucson, 1983.

Phillips, R. J. and C. T. Russell, Upper limit on the intrinsic magnetic field of Venus, J. Geophys. Res., 92, 2253-2263, 1987.

Rikitake, T., Oscillations of a system of disk dynamos, Proc. Cambridge Phil. Soc., 54, 89-105, 1958.

Rikitake, T., Non-dipole field and fluid motion in the Earth's core, J. Geomag. Geoelectr., 19, $129-142,1967$. 
Russell, C. T., Re-evaluating Bode's law of planetary magnetism, Nature, 272, 147-148, 1978.

Russell, C. T., Scaling law test and two predictions of planetary magnetic moments, Nature, 281, 552-553, 1979.

SHIMIZU, M. and Y. HonkurA, Fluctuations of magnetic fields in coupled-disk dynamo models and their implications for geomagnetic secular variations, J. Geomag. Geoelectr., 38, 611-632, 1986.

STEenbeck, M., F. KRAUSE, and K. H. RÄDLER, A calculation of the mean electromotive force in an electrically conducting fluid in turbulent motion, under the influence of Coriolis forces, Z. Naturforsch., 21a, 369-376, 1966.

Steenbeck, M. and F. Krause, Zur Dynamotheorie stellarer und planetarer Magnetfelder I. Berechnung sonnenähnlicher Wechselfeldgeneratoren, Astron. Nachr. Bd, 291 H.2, 49-83, 1969.

Stevenson, D., Interiors of the giant planets, Ann. Rev. Earth and Plan. Sci., 10, 257-295, 1982.

Stevenson, D., Planetary magnetic fields, Rep. Prog. Phys., 46, 555-620, 1983.

Tserklevich, A. L., Yu. P. Deineka, and G. A. Meshcheryakov, Gravitatsionnoe pole i raspredelenie plotnosti vnutri Marka (Gravity field and density distribution in the Martian interior), Geofiz. Zh., 5, 34-44, 1983.

Voorhies, C. V., Steady flows at the top of Earth's core derived from geomagnetic field models, J. Geophys. Res., 91, 12,444-12,466, 1986. 